\title{
Robust adaptive array beamforming with random error in cycle frequency
}

\author{
Y.-T.Lee and J.-H.Lee
}

\begin{abstract}
By exploiting the cyclostationary properties, the SCORE algorithms presented by Agee et al. (1990) have been shown to be effective in performing adaptive beamforming without requiring the direction vector of the desired signal. However, these algorithms suffer from severe performance degradation in the presence of a random error in cycle frequency. The authors first establish a statistical model of the cyclic correlation matrix when the random error exists. According to this statistical model, two robust methods based on the SCORE algorithms are developed to achieve robust adaptive beamforming against random error. Analytical formulas are then derived for evaluating the performance of the proposed methods. Several simulation examples are also presented for confirming the theoretical analysis and showing the effectiveness of the proposed methods.
\end{abstract}

\section{Introduction}

Cyclostationarity [1], which is a statistical property exhibited by most man-made communication signals, corresponds to the underlying periodicity arising from carrier frequencies or baud rates. Recently, a class of spectral selfcoherence restoral (SCORE) algorithms has been presented in [2] to deal with blind adaptive beamforming for cyclostationary signals. However, these algorithms suffer from server performance degradation in the presence of eycle frequency error (CFE). Some previous work on the problem has been reported in [4-8]. A statistical analysis presented in $[4,5]$ shows the cycle leakage through a sinc function due to finite data. On the other hand, two approaches, namely multi-cyclic MUSIC and adaptive- $\alpha$ cyclic MUSIC, have been presented in [6] to deal with performance degradation due to CFE. However, they cannot tackle the problem efficiently. Variations of the adaptive- $\alpha$ cyclic MUSIC are explored in [7]. Based on the concept of subintervals, only the cyclic correlation matrices of subintervals are required to reduce the memory requirements and the sensitivity of cyclic MUSIC to CFE. Recently, an efficient technique for coping with the problem of nonrandom CFE in cyclostationarity-exploiting adaptive array beamforming was developed [8]. Robust adaptive beamforming is achieved by appropriately estimating the actual cycle frequency.

In this paper, two of the SCORE algorithms presented in [2], namely the least-square SCORE (LS-SCORE) and cross-SCORE algorithms, are considered in the presence of random CFE. Based on the central limit theorem, the cyclic correlation matrix with random CFE is equivalent to

(C) IEE, 2001

IEE Proceedings online no. 20010425

DOI: 10.1049/ip-rsn:20010425

Paper first received 21st February 2000 and in final revised form 19th March 2001

The authors are with the Department of Electrical Engineering, Building 2, National Taiwan University, Taipei 106, Taiwan, Republic of China

IEE Proc.-Radar, Sonar Navig., Vol. I48, No, 4, August 2001 the cyclic correlation matrix computed by using the actual cycle frequency plus a Gaussian random error matrix when the random CFE is an independent sequence. By making use of the ideas originally developed in [9], where two approaches for curing the performance degradation of conventional adaptive beamforming with random steering vector error were presented, two methods based on the LS-SCORE and cross-SCORE algorithms are developed to alleviate the performance degradation due to random CFE. Then, analytic formulas are derived for evaluating the performance of the proposed methods.

\section{Adaptive beamforming using cyclostationarity}

\subsection{Signal cyclostationarity}

For a signal $s(t)$, the cyclic correlation function and conjugate cyclic correlation function are defined as the following infinite-time averages:

$$
r_{s s}(f, \tau)=\left\langle s(t+\tau / 2) s^{*}(t-\tau / 2) e^{-j 2 \pi j i}\right\rangle_{\infty}
$$

and

$$
r_{s s^{*}}(f, \tau)=\left\langle s(t+\tau / 2) s(t-\tau / 2) e^{-j 2 \pi f t}\right\rangle_{\infty}
$$

respectively, where the superscript ' $*$ ' denotes the complex conjugate. $s(t)$ is then said to be cyclostationary if $r_{s s}(f, \tau)$ or $r_{s s^{*}}(f, \tau)$ does not equal zero at some time delay $\tau$ and cycle frequency (CF) $f \neq 0$. Let $x(t)$ denote the data vector received by an array. The cyclic correlation matrix and conjugate cyclic correlation matrix are defined by

$$
\boldsymbol{R}_{x x}(f, \tau)=\left\langle\boldsymbol{x}(t+\tau / 2) \boldsymbol{x}^{H}(t-\tau / 2) e^{-j 2 \pi / t}\right\rangle_{\infty}
$$

and

$$
\boldsymbol{R}_{x x^{*}}(f, \tau)=\left\langle\boldsymbol{x}(t+\tau / 2) \boldsymbol{x}^{T}(t-\tau / 2) e^{-j 2 \pi f t}\right\rangle_{\infty}
$$

respectively, where the superscript ' $H$ ' denotes the conjugate transpose and ' $T$ ' the transpose. 


\subsection{SCORE algorithms}

Consider an $M$-element array excited by a SOI (signal of interest), $J$ interferers, and spatially white noise. The received array data vector $\boldsymbol{x}(t)$ is then given by

$$
\boldsymbol{x}(t)=s(t) \boldsymbol{a}_{d}+\sum_{j=1}^{J} s_{j}(t) \boldsymbol{a}_{j}+\boldsymbol{n}(t)=s(t) \boldsymbol{a}_{d}+\boldsymbol{i}(t)
$$

where $s(t)$ and $s_{j}(t)$ denote the waveforms of SOI and the $j$ th interferer, $\boldsymbol{a}_{d}$ and $\boldsymbol{a}_{j}$ are the direction vectors of the SOI and the $j$ th interferer, respectively, and $n(t)$ is the noise vector. The array output is then given by $y(t)=\boldsymbol{w}^{I I} \boldsymbol{x}(t)$, where $\boldsymbol{w}$ denotes the weight vector.

Assume that $s(t)$ is cyclostationary and has a $\mathrm{CF}$ equal to $\alpha$, but $i(t)$ includes all signals not of interest (SNOIs) and noise and is temporally uncorrelated with $s(t)$. Based on the LS-SCORE algorithm of [2], a cost function is defined as follows:

$$
F_{l s}(w ; c)=\left\langle|y(t)-r(t)|^{2}\right\rangle_{T}
$$

where the reference signal $r(t)$ is given by $r(t)=c^{H} \boldsymbol{x}(t-\tau) e^{j 2 \pi a t}$ and $\langle\cdot\rangle_{T}$ denotes the average over the time interval $[0, T] . c$ is a control vector and is fixed for the LS-SCORE algorithm. The optimal weight vector $\boldsymbol{w}_{l}$ minimising eqn. 6 is given by

$$
\hat{\boldsymbol{w}}_{l}=\hat{\boldsymbol{R}}_{x x}^{-1} \hat{\boldsymbol{r}}_{x r}(\alpha)
$$

where $\hat{\boldsymbol{R}}_{x x}=\left\langle\boldsymbol{x}(t) \boldsymbol{x}^{I I}(t)\right\rangle_{T}$ and $\hat{\boldsymbol{r}}_{r r}(\alpha)=\left\langle\boldsymbol{x}(t) r^{*}(t)\right\rangle_{T}$ are the sample autocorrelation matrix of $x(t)$ and the crosscorrelation vector of $\boldsymbol{x}(t)$ and $r(t)$ computed over $[0, T]$, respectively.

To improve the convergence speed of the LS-SCORE algorithm, the cross-SCORE algorithm is proposed by [2], based on maximising the correlation coefficient, which is given by

$$
\tilde{F}_{c s}(\boldsymbol{w} ; c)=\frac{\left|\boldsymbol{w}^{H} \hat{\boldsymbol{R}}_{x u} \boldsymbol{c}\right|^{2}}{\left(\boldsymbol{w}^{H} \hat{\boldsymbol{R}}_{x x} \boldsymbol{w}\right)\left(\boldsymbol{c}^{H} \hat{\boldsymbol{R}}_{u t u} \boldsymbol{c}\right)}
$$

between $y(t)$ and $r(t)$, where $\boldsymbol{u}(t)=\boldsymbol{x}(t-\tau) \boldsymbol{e}^{j 2 \pi \alpha t}$ is the control signal. It is easy to show that the optimal weight and control vectors maximising eqn. 8 are the dominant eigenvectors of

$$
\lambda \hat{\boldsymbol{R}}_{x x} \hat{\boldsymbol{w}}_{c}=\hat{\boldsymbol{R}}_{x u} \hat{\boldsymbol{R}}_{u u}^{-1} \hat{\boldsymbol{R}}_{u x} \hat{\boldsymbol{w}}_{c} \quad \text { and } \quad \lambda \hat{\boldsymbol{R}}_{u u} \hat{\boldsymbol{c}}_{c}=\hat{\boldsymbol{R}}_{u x} \hat{\boldsymbol{R}}_{x x}^{-1} \hat{\boldsymbol{R}}_{x u} \hat{\boldsymbol{c}}_{c}
$$

respectively.

\section{Proposed methods}

\subsection{Statistical model}

Assume that $f=\alpha+\Delta \alpha_{p}$, where $f$ and $\alpha$ are the presumed $\mathrm{CF}$ and the actual $\mathrm{CF}$, respectively, $\Delta \alpha_{n}$ represents the random cycle frequency error (CFE) at the time instant $n T_{s}$, and $T_{s}$ is the sampling interval. Substituting $t=n T_{s}$ into eqn. 3 , we have the discrete-time version of the cyclic correlation matrix as

$$
\begin{aligned}
\boldsymbol{R}_{x x}(f, \tau) & =\lim _{N \rightarrow \infty} \frac{1}{N} \sum_{n=1}^{N} \boldsymbol{x}(n) \boldsymbol{x}^{H}(n-\tau) e^{-j 2 \pi\left(\alpha+\Delta \alpha_{n}\right) n T_{s}} \\
& =\boldsymbol{R}_{x x}(\alpha, \tau)+\boldsymbol{R}_{e}(f, \tau)
\end{aligned}
$$

where $\boldsymbol{R}_{e}(f, \tau)$ represents the corresponding error matrix equal to $\lim _{N \rightarrow \infty}(1 / N) \sum_{n=1}^{N}\left(e^{-j 2 \pi \Delta \alpha_{n} n T_{s}}-1\right) D_{x}(n, \tau)$ and $\boldsymbol{D}_{x}(n, \tau)=\boldsymbol{x}(n) \boldsymbol{x}^{H}(n-\tau) e^{-j 2 \pi \alpha n T_{s}}$ is independent of $\Delta x_{n}$. Let $\left\{\Delta \alpha_{n}, n=1,2, \ldots\right\}$ be an independent random sequence; the random sequence $\left\{\left(e^{-j 2 \pi \Delta \alpha_{n} n T_{s}}-1\right), n=1\right.$, $2, \ldots\}$ is thus independent. Then, $\boldsymbol{R}_{e}(f, \tau)$ equals the sum of infinite independent random matrices and becomes a Gaussian random matrix according to the central limit theorem shown by $([10]$, p. 15). As a result, we conclude that when $\mathrm{CFE}$ is an independent random sequence, the resulting cyclic correlation matrix is equal to the cyclic correlation matrix computed by using the actual $\mathrm{CF}$ plus a Gaussian random error matrix.

\subsection{Robust methods based on LS-SCORE algorithm}

From eqn. 10, the weight vector shown by eqn. 7 can be expressed as

$$
\boldsymbol{w}_{l}=\boldsymbol{R}_{x x}^{-1} \boldsymbol{R}_{x x}(f, \tau) \boldsymbol{c}=\boldsymbol{R}_{x x}^{-1}\left\{\boldsymbol{R}_{x x}(\alpha, \tau) \boldsymbol{c}+\boldsymbol{R}_{e}(f, \tau) \boldsymbol{c}\right\}
$$

when $T$ approaches infinity. Let $\boldsymbol{R}_{e}(f, \tau)$ have zero mean. Then, the vector $\boldsymbol{a}_{e}=\boldsymbol{R}_{e}(f, \tau) \boldsymbol{c}$ is a zero-mean Gaussian random vector since $c$ is a fixed vector. Hence, the loglikelihood function corresponding to $a_{e}$ is given by

$$
\begin{aligned}
f_{\mathrm{L}}= & -\boldsymbol{a}_{e}^{H} \boldsymbol{C}^{-1} \boldsymbol{a}_{e}=-\boldsymbol{c}^{H}\left(\boldsymbol{R}_{x x}(\alpha, \tau)\right. \\
& \left.-\boldsymbol{R}_{x x}(f, \tau)\right) \boldsymbol{C}^{-1}\left(\boldsymbol{R}_{x x}(\alpha, \tau)-\boldsymbol{R}_{x x}(f, \tau)\right) \boldsymbol{c}
\end{aligned}
$$

wherc $C$ denotes the covariance matrix of $\boldsymbol{a}_{e}$

Accordingly, the role of $\boldsymbol{a}_{e}$ in eqn. 11 can be viewed as the resulting steering crror vector due to the random CFE. Using the first method of [9], we define a cost function related to $\boldsymbol{a}_{e}$ as

$$
J(\boldsymbol{a})=\boldsymbol{a}^{H} \boldsymbol{R}_{x x}^{-1} \boldsymbol{a}+k\left(\boldsymbol{a}-\boldsymbol{R}_{x x}(f, \tau) c\right)^{H} \boldsymbol{C}^{-1}\left(\boldsymbol{a}-\boldsymbol{R}_{x x}(f, \tau) \boldsymbol{c}\right)
$$

where the first term is the inverse of array output power corresponding to the constraint vector $\boldsymbol{a}, \boldsymbol{R}_{x x}$ is the ensemble autocorrelation matrix of $\boldsymbol{x}(t)$, and $k$ is a positive parameter. We obtain the optimal $a_{o}$ and $w_{l 1}$ for minimising eqn. 13 as

$$
\boldsymbol{a}_{o}=\left(\boldsymbol{I}+\frac{1}{k} \boldsymbol{C} \boldsymbol{R}_{x x}^{-1}\right)^{-1} \boldsymbol{R}_{x x}(f, \tau) \boldsymbol{c}
$$

and

$$
\boldsymbol{w}_{l 1}=\left(\boldsymbol{R}_{x x}+\frac{1}{k} \boldsymbol{C}\right)^{-1} \boldsymbol{R}_{x x}(f, \tau) c
$$

Next, performing the cigendecomposition of $\boldsymbol{R}_{x x}$, we have $\boldsymbol{R}_{x x}=\sum_{i=1}^{M} \hat{\lambda}_{i} \boldsymbol{e}_{i} \boldsymbol{e}_{i}^{H}$, where $\lambda_{1} \geq \cdots \geq \lambda_{J+1}>\lambda_{J+2}=\cdots=$ $\lambda_{M}=\sigma_{n}^{2}$ are the eigenvalues of $\boldsymbol{R}_{x x}$ in descending order, $\sigma_{n}^{2}$ is the variance of the noise, and $e_{i}, i=1, \ldots, M$ are the corresponding eigenvectors. The subspaces spanned by $\boldsymbol{E}_{s}=\left\{\boldsymbol{e}_{1}, \ldots, \boldsymbol{e}_{J+1}\right\}$ and $\boldsymbol{E}_{n}=\left\{\boldsymbol{e}_{J+2}, \ldots, \boldsymbol{e}_{M}\right\}$ are called the signal subspace and noise subspace, respectively. Hence, $\boldsymbol{R}_{x x}$ can be rewritten as

$$
\boldsymbol{R}_{x x}=\boldsymbol{E}_{s} \boldsymbol{\Lambda}_{s} \boldsymbol{E}_{s}^{H}+\boldsymbol{E}_{n} \boldsymbol{\Lambda}_{n} \boldsymbol{E}_{n}^{H}
$$

where $\boldsymbol{\Lambda}_{s}=\operatorname{diag}\left\{\lambda_{1}, \ldots, \lambda_{J+1}\right\}$ and $\boldsymbol{\Lambda}_{n}=\sigma_{n}^{2} \boldsymbol{I}$. Since $\boldsymbol{R}_{x x}(\alpha, \tau) \boldsymbol{c}=r_{s s}(\alpha, \tau)\left(\boldsymbol{a}_{d}^{H} c\right) \boldsymbol{a}_{d}$ lies in the signal subspace, we use the second method of [9] and define a cost function as

$J(\boldsymbol{a})=\boldsymbol{a}^{I H} \boldsymbol{E}_{n} \boldsymbol{E}_{n}^{H} \boldsymbol{a}+k\left(\boldsymbol{a}-\boldsymbol{R}_{x x}(f, \tau) \boldsymbol{c}\right)^{H} \boldsymbol{C}^{-1}\left(\boldsymbol{a}-\boldsymbol{R}_{x x}(f, \tau) \boldsymbol{c}\right)$

The optimal $\boldsymbol{a}_{O}$ and $\boldsymbol{w}_{l 2}$ minimising eqn. 17 can be easily obtained as

$$
\boldsymbol{a}_{o}=\left(\boldsymbol{I}+\frac{1}{k} \boldsymbol{C} \boldsymbol{E}_{n} \boldsymbol{E}_{n}^{H}\right)^{-1} \boldsymbol{R}_{x x}(f, \tau) \boldsymbol{c}
$$


and

$$
\boldsymbol{w}_{l 2}=\left(\boldsymbol{E}_{s} \boldsymbol{\Lambda}_{s} \boldsymbol{E}_{s}^{I l}+\left(\boldsymbol{I}+\frac{1}{k} \boldsymbol{C}\right) \boldsymbol{E}_{n} \boldsymbol{\Lambda}_{n} \boldsymbol{E}_{n}^{I I}\right)^{-1} \boldsymbol{R}_{x x}(f, \tau) \boldsymbol{c}
$$

\subsection{Robust methods based on cross-SCORE algorithm}

Since $\boldsymbol{R}_{x u}=\boldsymbol{R}_{x x}(f, \tau) e^{-j \pi f \tau}$ and $\boldsymbol{R}_{u u}^{-1}=\boldsymbol{R}_{x x}^{-1}$, we obtain from eqns. 9 and 10 that the weight vector $\boldsymbol{w}_{c}$ of the original cross-SCORE algorithm equals the dominant cigenvector of

$$
\begin{aligned}
\lambda \boldsymbol{w}_{c}= & \boldsymbol{R}_{x x}^{-1} \boldsymbol{R}_{x x}(f, \tau) \boldsymbol{R}_{x x}^{-1} \boldsymbol{R}_{x x}^{H}(f, \tau) \boldsymbol{w}_{c} \\
= & \boldsymbol{R}_{x x}^{-1} \boldsymbol{R}_{x x}(\alpha, \tau) \boldsymbol{R}_{x x}^{-1} \boldsymbol{R}_{x x}^{H}(\alpha, \tau) \boldsymbol{w}_{c} \\
& +\boldsymbol{R}_{x x}^{-1} \boldsymbol{R}_{x x}(\alpha, \tau) \boldsymbol{R}_{x x}^{-1} \boldsymbol{R}_{e}^{H}(f, \tau) \boldsymbol{w}_{c} \\
& +\boldsymbol{R}_{x x}^{-1} \boldsymbol{R}_{e}(f, \tau) \boldsymbol{R}_{x x}^{-1} \boldsymbol{R}_{x x}(\alpha, \tau) \boldsymbol{w}_{c} \\
& +\boldsymbol{R}_{x x}^{-1} \boldsymbol{R}_{e}(f, \tau) \boldsymbol{R}_{x x}^{-1} \boldsymbol{R}_{e}^{I}(f, \tau) \boldsymbol{w}_{c}
\end{aligned}
$$

when $T$ approaches infinity. Substituting $\boldsymbol{R}_{x x}(\alpha, \tau)=$ $r_{s s}(\alpha, \tau) a_{d} a_{d}^{H}$ into eqn. 20 and performing some algebraic manipulations, we have

$$
\begin{gathered}
\boldsymbol{R}_{x x}^{-1} \boldsymbol{R}_{x x}(\alpha, \tau) \boldsymbol{R}_{x x}^{-1} \boldsymbol{R}_{x x}^{H}(\alpha, \tau) \boldsymbol{w}_{c}= \\
\underbrace{\left\{\left|r_{s s}(\alpha, \tau)\right|^{2}\left(\boldsymbol{a}_{d}^{H} \boldsymbol{R}_{x x}^{-1} \boldsymbol{a}_{d}\right)\left(\boldsymbol{a}_{d}^{H} \boldsymbol{w}_{c}\right)\right\}}_{\gamma_{1}} \boldsymbol{R}_{x x}^{-1} \boldsymbol{a}_{d} \\
\boldsymbol{R}_{x x}^{-1} \boldsymbol{R}_{x x}(\alpha, \tau) \boldsymbol{R}_{x x}^{-1} \underbrace{\boldsymbol{R}_{e}^{H}(f, \tau) \boldsymbol{w}_{c}=}_{\gamma_{2}} \\
\underbrace{\boldsymbol{r}_{x x}^{-1} \boldsymbol{a}_{d}}_{\left.\gamma_{s x}(\alpha, \tau)\left(\boldsymbol{a}_{d}^{H} \boldsymbol{R}_{x x}^{-1} \boldsymbol{R}_{e}(f, \tau) \boldsymbol{w}_{c}\right)\right\}} \\
\boldsymbol{R}_{x x}^{-1} \boldsymbol{R}_{e}(f, \tau) \boldsymbol{R}_{x x}^{-1} \boldsymbol{R}_{x x}^{H}(\alpha, \tau) \boldsymbol{w}_{c}=\underbrace{\left\{r_{s s}^{*}(\alpha, \tau)\left(\boldsymbol{a}_{d}^{H} \boldsymbol{w}_{c}\right)\right\}}_{\gamma_{3 x}} \boldsymbol{R}_{x x}^{-1} \boldsymbol{a}_{e 1} \\
\boldsymbol{R}_{x x}^{-1} \boldsymbol{R}_{e}(f, \tau) \boldsymbol{R}_{x x}^{-1} \boldsymbol{R}_{e}^{H}(f, \tau) \boldsymbol{w}_{c}=\boldsymbol{R}_{x x}^{-1} \boldsymbol{a}_{e 2}
\end{gathered}
$$

where $\boldsymbol{a}_{e \mathrm{l}}=\boldsymbol{R}_{e}(f, \tau) \boldsymbol{R}_{x x}^{-1} \boldsymbol{a}_{d}$ is a Gaussian random vector, and $\boldsymbol{a}_{e 2}=\boldsymbol{R}_{e}(f, \tau) \boldsymbol{R}_{x x}^{-1} \boldsymbol{R}_{e}^{H}(f, \tau) \boldsymbol{w}_{c}$ is not a Gaussian random vector due to the nonlinear operation of $\boldsymbol{R}_{e}(f, \tau)$. Let the elements of $\boldsymbol{R}_{e}(f, \tau)$ be independent identically distributed (i.i.d.) white Gaussian random variables with zero mean and variance $\sigma_{e}^{2}$. Then, eqn. 23 becomes a white Gaussian random vector. Assume that $\sigma_{e}^{2}$ is small enough. The term of eqn. 24 is negligible in comparison with the other terms of eqns. 21, 22 and 23. Accordingly, we can rewrite eqn. 20 as

$$
\boldsymbol{w}_{c} \approx \boldsymbol{R}_{x x}^{-1}\left\{\frac{\gamma_{1}+\gamma_{2}}{\lambda} \boldsymbol{a}_{d}+\frac{\gamma_{3}}{\lambda} \boldsymbol{a}_{e 1}\right\}=\mu \boldsymbol{R}_{x x}^{-1}\left(\boldsymbol{a}_{d}+\boldsymbol{a}_{e}\right)
$$

where

$$
\mu=\frac{\gamma_{1}+\gamma_{2}}{2} \text { and } \boldsymbol{a}_{e}=\frac{\gamma_{3}}{\gamma_{1}+\gamma_{2}} \boldsymbol{a}_{e 1}
$$

is a zero-mean white Gaussian random vector. We observe from eqns. 11 and 25 that the weight vectors obtained by using the LS-SCORE and the cross-SCORE algorithms have the similar form in the presence of random CFE. Letting the covariance matrix of $\boldsymbol{a}_{c}$ in eqn. 25 be $\boldsymbol{C}=\sigma_{c}^{2} \boldsymbol{I}$, we note from eqns. 15 and 19 that based on the crossSCORE algorithm it is appropriate to set the optimal weight vector equal to the dominant eigenvector of

$$
\lambda \boldsymbol{w}_{c}=\boldsymbol{R}_{z z}^{-1} \boldsymbol{R}_{x x}(f, \tau) \boldsymbol{R}_{z z}^{-1} \boldsymbol{R}_{x x}^{H}(f, \tau) \boldsymbol{w}_{c}
$$

where

$$
\boldsymbol{R}_{z z}^{-1}=\left\{\begin{array}{l}
\left(\boldsymbol{R}_{x x}+\frac{\sigma_{c}^{2}}{k} \boldsymbol{I}\right)^{-1} \text { for the first method } \\
\boldsymbol{E}_{s} \boldsymbol{\Lambda}_{s}^{-1} \boldsymbol{E}_{s}^{H}+\frac{k}{k+\sigma_{c}^{2}} \boldsymbol{E}_{n} \boldsymbol{\Lambda}_{n}^{-1} \boldsymbol{E}_{n}^{H} \\
\text { for the second method }
\end{array}\right.
$$

\section{Theoretical analysis}

Here, we evaluate the performance in terms of the array output signal-to-interference plus noise power ratio (SINR) for each of the methods proposed above. Consider an $M$ element uniform linear array (ULA) excited by a SOI, an interferer, and additive white Gaussian noise (AWGN). Substituting $\boldsymbol{R}_{x x}(\alpha, \tau)=r_{s, s}(\alpha, \tau) \boldsymbol{a}_{d} \boldsymbol{a}_{d}^{H}$ and $\boldsymbol{C}=\sigma_{c}^{2} \boldsymbol{I}$ into eqns. 15 and 19 , we obtain that the optimal weight vector $\boldsymbol{w}_{l}$ equals $\boldsymbol{R}_{z z}^{-1} \boldsymbol{R}_{x x}(f, \tau) \boldsymbol{c}=\mu \boldsymbol{R}_{z z}^{-1}\left(\boldsymbol{a}_{d}+(\mathbf{1} / \mu) \boldsymbol{R}_{e}(f\right.$, $\tau) c$ ) for the proposed methods based on the LS-SCORE algorithm, where $\mu=r_{s s}(\alpha, \tau)\left(\boldsymbol{a}_{d}^{I} \boldsymbol{c}\right)$ and $\boldsymbol{R}_{z z}^{-1}$ is given by eqn. 27. In contrast, we have from eqns. 25 and 26 that $\boldsymbol{w}_{c} \approx \mu \boldsymbol{R}_{z z}^{-1}\left\{\boldsymbol{a}_{d}+\left[\gamma_{3} /\left(\gamma_{1}+\gamma_{2}\right)\right] \boldsymbol{a}_{e 1}\right\}$ when using the crossSCORE algorithm, where $\mu=\left(\gamma_{1}+\gamma_{2}\right) / \lambda$. Moreover, $\gamma_{1}, \gamma_{2}$ and $\gamma_{3}$ are given by eqns. 21,22 and 23 , respectively, except that $\boldsymbol{R}_{z z}^{-1}$ is used instead of $\boldsymbol{R}_{x y}^{-1}$. As a result, under the situation where the resulting steering error vector is white Gaussian, the optimal weight vector obtained by using the proposed methods developed based on either the LS-SCORE or cross-SCORE algorithms has a general expression

$$
\boldsymbol{w}_{e q}=\mu \boldsymbol{R}_{z z}^{-1}\left(\boldsymbol{a}_{d}+\sigma_{c} \boldsymbol{a}_{e}\right)
$$

where $\left|\boldsymbol{a}_{d}\right|^{2}=M$, and $\sigma_{c} \boldsymbol{a}_{\mathfrak{e}}$ denotes the corresponding white Gaussian steering error vector with mean zero and covariance matrix $\sigma_{c}^{2} \boldsymbol{I}$. We note that $\sigma_{c} \boldsymbol{a}_{e}$ is given by $(1 / \mu) \boldsymbol{R}_{e}(f, \tau) \boldsymbol{c}$ and $\left[\gamma_{3} /\left(\gamma_{1}+\gamma_{2}\right)\right] \boldsymbol{a}_{e 1}$ for the proposed methods based on the LS-SCORE algorithm and crossSCORE algorithm, respectively. Let $\sigma_{s}^{2}, \sigma_{j}^{2}$, and $\sigma_{n}^{2}$ represent the powers of SOI, interference and noise, respectively. From eqns. 16 and 27 , we can rewrite $\boldsymbol{R}_{z z}^{-1}$ as

$$
\boldsymbol{R}_{z z}^{-1}=\boldsymbol{E}_{s} \tilde{\boldsymbol{\Lambda}}_{s}^{-1} \boldsymbol{E}_{s}^{H}+\boldsymbol{E}_{n} \tilde{\boldsymbol{\Lambda}}_{n}^{-1} \boldsymbol{E}_{n}^{H}
$$

where

$$
\left[\tilde{\boldsymbol{\Lambda}}_{s}, \tilde{\boldsymbol{\Lambda}}_{n}\right]= \begin{cases}{\left[\boldsymbol{\Lambda}_{s}+\frac{\sigma_{c}^{2}}{k} \boldsymbol{I},\left(\sigma_{n}^{2}+\frac{\sigma_{c}^{2}}{k}\right) \boldsymbol{I}\right.} & \\ {\left[\boldsymbol{\Lambda}_{s}, \frac{k+\sigma_{c}^{2}}{k} \boldsymbol{\Lambda}_{n}\right]} & \text { for the first method } \\ & \text { for the second method }\end{cases}
$$

For deriving the output SINR for the proposed methods, we first rewrite $\boldsymbol{a}_{e}=c_{s} \boldsymbol{a}_{d l}+c_{j} \boldsymbol{a}_{j}+\boldsymbol{E}_{n} \boldsymbol{E}_{n}^{H} \boldsymbol{a}_{e}$. Due to the fact that $\boldsymbol{a}_{d}^{I I} \boldsymbol{E}_{n} \boldsymbol{E}_{n}^{H} \boldsymbol{a}_{e}=\boldsymbol{a}_{j}^{H} \boldsymbol{E}_{n} \boldsymbol{E}_{n}^{H} \boldsymbol{a}_{e}=0$, we have

$$
\left\{\begin{array}{l}
\boldsymbol{a}_{d}^{H} \boldsymbol{a}_{e}=M c_{s}+\left(\boldsymbol{a}_{d}^{H} \boldsymbol{a}_{j}\right) c_{j} \\
\boldsymbol{a}_{j}^{H} \boldsymbol{a}_{e}=\left(\boldsymbol{a}_{j}^{H} \boldsymbol{a}_{d}\right) c_{s}+M c_{j}
\end{array}\right.
$$

and hence

$$
c_{s}=\frac{M \boldsymbol{a}_{d}^{H} \boldsymbol{a}_{e}-\left(\boldsymbol{a}_{d}^{H} \boldsymbol{a}_{j}\right) \boldsymbol{a}_{j}^{H} \boldsymbol{a}_{e}}{M^{2}-\left|\boldsymbol{a}_{j}^{H} \boldsymbol{a}_{d}\right|^{2}} \text { and } c_{j}=\frac{M \boldsymbol{a}_{j}^{H} \boldsymbol{a}_{e}-\left(\boldsymbol{a}_{j}^{H} \boldsymbol{a}_{d}\right) \boldsymbol{a}_{d}^{H} \boldsymbol{a}_{e}}{M^{2}-\left|\boldsymbol{a}_{j}^{H} \boldsymbol{a}_{d}\right|^{2}}
$$


Moreover, because $\boldsymbol{a}_{j}^{H} \boldsymbol{R}_{x x}^{-1} \boldsymbol{a}_{d} \approx 0$, we have $\boldsymbol{a}_{j}^{H} \boldsymbol{E}_{s} \boldsymbol{\Lambda}_{s}^{-1} \boldsymbol{E}_{s}^{H} \boldsymbol{a}_{d} \approx 0$ and $\boldsymbol{a}_{j}^{H}\left\{\boldsymbol{R}_{x x}+\left(\sigma_{c}^{2} / k\right) \boldsymbol{I}^{-1} \boldsymbol{a}_{d} \approx 0\right.$ when $\sigma_{c}^{2} / k$ is small enough. Therefore, $\boldsymbol{a}_{j}^{H} \boldsymbol{E}_{s} \tilde{\boldsymbol{\Lambda}}_{s}^{-1} \boldsymbol{E}_{s}^{H} \boldsymbol{a}_{d} \approx 0$ for the first and second methods. On the other hand, $\boldsymbol{a}_{e}^{H} \boldsymbol{E}_{n} \boldsymbol{E}_{n}^{H} \boldsymbol{E}_{s} \tilde{\boldsymbol{\Lambda}}_{s}^{-1} \boldsymbol{E}_{s}^{H} \boldsymbol{a}_{d}=0$ and $\boldsymbol{a}_{e}^{H} \boldsymbol{E}_{n} \tilde{\boldsymbol{\Lambda}}_{n}^{-1} \boldsymbol{E}_{n}^{H} \boldsymbol{a}_{d d}=0$ due to the orthogonality of $\boldsymbol{E}_{s}$ and $\boldsymbol{E}_{n}$. Accordingly, we have

$$
\boldsymbol{a}_{e}^{H} \boldsymbol{E}_{s} \tilde{\boldsymbol{\Lambda}}_{s}^{-1} \boldsymbol{E}_{s}^{H} \boldsymbol{a}_{d} \approx c_{s}^{*} \boldsymbol{a}_{d}^{H} \boldsymbol{E}_{s} \tilde{\boldsymbol{\Lambda}}_{s}^{-1} \boldsymbol{E}_{s}^{H} \boldsymbol{a}_{d}
$$

and the output power gain of SOI is

$$
\begin{aligned}
P G_{s} & =\left|\boldsymbol{w}_{e q}^{H} \boldsymbol{a}_{d}\right|^{2} \\
& =|\mu|^{2}\left|\left(\boldsymbol{a}_{d}+\sigma_{c} \boldsymbol{a}_{e}\right)^{H}\left\{\boldsymbol{E}_{s} \tilde{\boldsymbol{\Lambda}}_{s}^{-1} \boldsymbol{E}_{s}^{H}+\boldsymbol{E}_{n} \tilde{\boldsymbol{\Lambda}}_{n}^{-1} \boldsymbol{E}_{n}^{H}\right\} \boldsymbol{a}_{d}\right|^{2} \\
& =|\mu|^{2}\left|\left(\boldsymbol{a}_{d}+\sigma_{c} \boldsymbol{a}_{e}\right)^{H} \boldsymbol{E}_{s} \tilde{\boldsymbol{\Lambda}}_{s}^{-1} \boldsymbol{E}_{s}^{H} \boldsymbol{a}_{d}\right|^{2} \\
& \approx|\mu|^{2}\left|1+\sigma_{c} c_{s}\right|^{2}\left(\boldsymbol{a}_{d}^{H} \boldsymbol{E}_{s} \tilde{\boldsymbol{\Lambda}}_{s}^{-1} \boldsymbol{E}_{s}^{H} \boldsymbol{a}_{d}\right)^{2}
\end{aligned}
$$

Similarly, because $\boldsymbol{a}_{e}^{H} \boldsymbol{E}_{n} \boldsymbol{E}_{n}^{H} \boldsymbol{E}_{s} \tilde{\boldsymbol{\Lambda}}_{s}^{-1} \boldsymbol{E}_{s}^{H} \boldsymbol{a}_{j}=\mathbf{0} \quad$ and $\boldsymbol{a}_{e}^{I I} \boldsymbol{E}_{n} \tilde{\boldsymbol{\Lambda}}_{n}^{-1} \boldsymbol{E}_{n}^{H} \boldsymbol{a}_{j}=0$, the output power gain of interference can be expressed by

$$
\begin{aligned}
P G_{j} & =\left|\boldsymbol{w}_{e q}^{H} \boldsymbol{a}_{j}\right|^{2}=|\mu|^{2}\left|\left(\boldsymbol{a}_{d}+\sigma_{c} \boldsymbol{a}_{e}\right)^{H} \boldsymbol{E}_{s} \tilde{\boldsymbol{\Lambda}}_{s}^{-1} \boldsymbol{E}_{s}^{H} \boldsymbol{a}_{j}\right|^{2} \\
& =\left|\mu \sigma_{c}\right|^{2}\left|\boldsymbol{a}_{e}^{H} \boldsymbol{E}_{s} \tilde{\boldsymbol{\Lambda}}_{s}^{-1} \boldsymbol{E}_{s}^{H} \boldsymbol{a}_{j}\right|^{2} \\
& \approx\left|\mu \sigma_{c} c_{j}\right|^{2}\left(\boldsymbol{a}_{j}^{H} \boldsymbol{E}_{s} \tilde{\boldsymbol{\Lambda}}_{s}^{-1} \boldsymbol{E}_{s}^{H} \boldsymbol{a}_{j}\right)^{2}
\end{aligned}
$$

Moreover, the output power gain of noise is given by

$$
\begin{aligned}
P G_{n}= & \boldsymbol{w}_{e q}^{H} \boldsymbol{w}_{e q}=|\mu|^{2}\left(\boldsymbol{a}_{d}+\sigma_{c} \boldsymbol{a}_{e}\right)^{H} \boldsymbol{R}_{z z}^{-2}\left(\boldsymbol{a}_{d}+\sigma_{c} \boldsymbol{a}_{e}\right) \\
= & |\mu|^{2}\left\{\boldsymbol{a}_{d}^{H} \boldsymbol{R}_{z z}^{-2} \boldsymbol{a}_{d}+\sigma_{c} \boldsymbol{a}_{e}^{H} \boldsymbol{R}_{z z}^{-2} \boldsymbol{a}_{d}\right. \\
& \left.+\sigma_{c} \boldsymbol{a}_{d}^{H} \boldsymbol{R}_{z z}^{-2} \boldsymbol{a}_{e}+\sigma_{c}^{2} \boldsymbol{a}_{e}^{H} \boldsymbol{R}_{z z}^{-2} \boldsymbol{a}_{e}\right\} \\
= & |\mu|^{2}\left\{\boldsymbol{a}_{d}^{H} \boldsymbol{E}_{s} \tilde{\boldsymbol{\Lambda}}_{s}^{-2} \boldsymbol{E}_{s}^{H} \boldsymbol{a}_{d}+\sigma_{c} \boldsymbol{a}_{e}^{H} \boldsymbol{E}_{s} \tilde{\boldsymbol{\Lambda}}_{s}^{-2} \boldsymbol{E}_{s}^{H} \boldsymbol{a}_{d}\right. \\
& \left.+\sigma_{c} \boldsymbol{a}_{d}^{H} \boldsymbol{E}_{s} \tilde{\boldsymbol{\Lambda}}_{s}^{-2} \boldsymbol{E}_{s}^{H} \boldsymbol{a}_{e}+\sigma_{c}^{2} \boldsymbol{a}_{e}^{H} \boldsymbol{R}_{z z}^{-2} \boldsymbol{a}_{e}\right\} \\
\approx & |\mu|^{2}\left\{\left(\boldsymbol{a}_{d}^{H} \boldsymbol{E}_{s} \tilde{\boldsymbol{\Lambda}}_{s}^{-2} \boldsymbol{E}_{s}^{H} \boldsymbol{a}_{d}\right)\left(1+\sigma_{c} c_{s}+\sigma_{c} c_{s}^{*}\right)\right. \\
& \left.+\sigma_{c}^{2} \boldsymbol{a}_{e}^{H} \boldsymbol{R}_{z z}^{-2} \boldsymbol{a}_{e}\right\}
\end{aligned}
$$

where $\boldsymbol{R}_{z z}^{-2}=\boldsymbol{E}_{s} \tilde{\boldsymbol{\Lambda}}_{s}^{-2} \boldsymbol{E}_{s}^{H}+\boldsymbol{E}_{n} \tilde{\boldsymbol{\Lambda}}_{n}^{-2} \boldsymbol{E}_{n}^{H}$ and $\boldsymbol{a}_{e}^{H} \boldsymbol{E}_{s} \tilde{\boldsymbol{\Lambda}}_{s}^{-2} \boldsymbol{E}_{s}^{H} \boldsymbol{a}_{d} \approx$ $c_{s}^{*} \boldsymbol{a}_{d}^{H} \boldsymbol{E}_{s} \tilde{\boldsymbol{\Lambda}}_{s}^{-2} \boldsymbol{E}_{s}^{H} \boldsymbol{a}_{d}$ are obtained from equns. 29 and 32 , respectively.

Next, we evaluate $\boldsymbol{a}_{d}^{H} \boldsymbol{E}_{s} \tilde{\boldsymbol{\Lambda}}_{s}^{-p} \boldsymbol{E}_{s}^{H} \boldsymbol{a}_{d}$ and $\boldsymbol{a}_{j}^{H} \boldsymbol{E}_{s} \tilde{\boldsymbol{\Lambda}}_{s}^{-p} \boldsymbol{E}_{s}^{H} \boldsymbol{a}_{j}$ for $p=1$ and 2 . Consider the case that there only exists a SOI and background noise; it is easy to show that $\boldsymbol{E}_{s}=\boldsymbol{e}_{\max } \propto \boldsymbol{a}_{d} / \sqrt{ } M$ and $\tilde{\boldsymbol{\Lambda}}_{s}=\xi_{s}=M \sigma_{s}^{2}+\sigma_{n}^{2}+\sigma_{c}^{2} / k$ for the first method. Hence, we have $\boldsymbol{a}_{d}^{H} \boldsymbol{E}_{s} \tilde{\boldsymbol{\Lambda}}_{s}^{-p} \boldsymbol{E}_{s}^{H} \boldsymbol{a}_{d} \approx M \xi_{s}^{-p}$ for the first method. When there exists an additional interferer, we note that $\boldsymbol{a}_{d}^{H} \boldsymbol{E}_{s} \tilde{\boldsymbol{\Lambda}}_{s}^{-p} \boldsymbol{E}_{s}^{H} \boldsymbol{a}_{d} \approx \boldsymbol{M} \xi_{s}^{-p}$ is also valid if $\left|\boldsymbol{a}_{d}^{H} \boldsymbol{a}_{j}\right| \ll M$. Similarly, we have $\boldsymbol{a}_{j}^{H} \boldsymbol{E}_{s} \tilde{\boldsymbol{\Lambda}}_{s}^{-p} \boldsymbol{E}_{s}^{H} \boldsymbol{a}_{j} \approx$ $M \xi_{j}^{-p}$, where $\xi_{j}=M \sigma_{j}^{2}+\sigma_{n}^{2}+\sigma_{c}^{2} / k$. Accordingly, the output SINR obtained by using eqn. 28 for the first method, based on either the LS-SCORE or the crossSCORE algorithms, is given by

$$
\begin{aligned}
& S I N R_{1}=\frac{P G_{s} \times \sigma_{s}^{2}}{P G_{j} \times \sigma_{j}^{2}+P G_{n} \times \sigma_{n}^{2}}= \\
& \frac{M^{2} \xi_{s}^{-2}\left|1+\sigma_{c} c_{s}\right|^{2} S N R}{\sigma_{c}^{2}\left|c_{j}\right|^{2} M^{2} \xi_{j}^{-2} I N R+M \xi_{s}^{-2}\left(1+\sigma_{c} c_{s}+\sigma_{c} c_{s}^{*}\right)+\sigma_{c}^{2} a_{e}^{H} \boldsymbol{R}_{z z}^{-2} \boldsymbol{a}_{e}}
\end{aligned}
$$

where $S N R=\sigma_{s}^{2} / \sigma_{n}^{2}$ and $I N R=\sigma_{j}^{2} / \sigma_{n}^{2}$. From eqn. 44 in the Appendix, the expectation of $S I N R_{1}$ is approximately given by

$$
\begin{aligned}
& E\left\{S I N R_{1}\right\} \\
& \begin{aligned}
= & \frac{M^{2} \xi_{s}^{-2} E\left\{\left|1+\sigma_{c} c_{s}\right|^{2}\right\} S N R}{\sigma_{c}^{2} E\left\{\left|c_{j}\right|^{2}\right\} M^{2} \xi_{j}^{-2} I N R} \\
& +M \xi_{s}^{-2} E\left\{\left(1+\sigma_{c} c_{s}+\sigma_{c} c_{s}^{*}\right)\right\}+\sigma_{c}^{2} E\left\{\boldsymbol{a}_{e}^{H} \boldsymbol{R}_{z z}^{-2} \boldsymbol{a}_{e}\right\}
\end{aligned} \\
& =M^{2} \xi_{s}^{-2} S N R /\left[M \xi_{s}^{-2}+\left(M^{2} I N R+M\right) \xi_{j}^{-2}\right.
\end{aligned}
$$

Similarly, we have $\boldsymbol{a}_{d}^{H} \boldsymbol{E}_{s} \tilde{\boldsymbol{\Lambda}}_{s}^{-p} \boldsymbol{E}_{s}^{H} \boldsymbol{a}_{d}=M \rho_{s}^{-p}$ and $\boldsymbol{a}_{j}^{H} \boldsymbol{E}_{s} \tilde{\boldsymbol{\Lambda}}_{s}^{-p} \boldsymbol{E}_{s}^{H} \boldsymbol{a}_{j}=M \rho_{j}^{-p}$ for the second method, where $\rho_{s}=M \sigma_{s}^{2}+\sigma_{n}^{2}$ and $\rho_{j}=M \sigma_{j}^{2}+\sigma_{n}^{2}$. From eqn. 45 , the output SINR and the expectation of the output SINR by
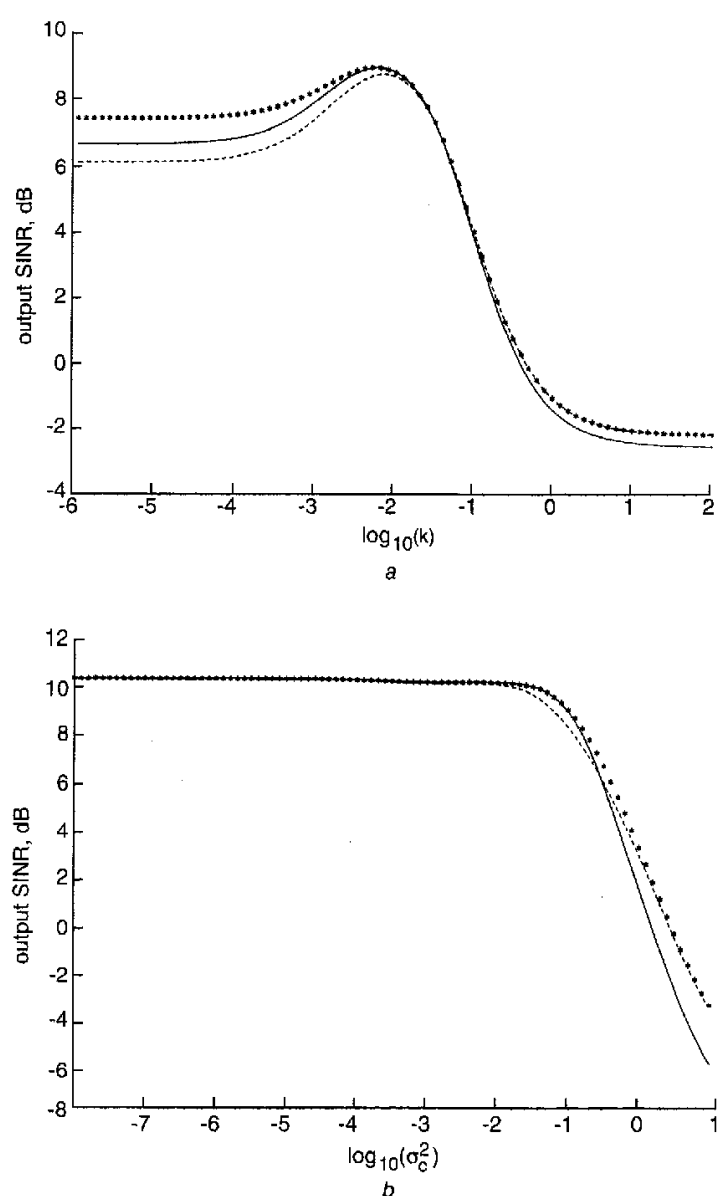

Fig. 1 Output SINR against logarithmic values of $k$ and $\sigma_{c}^{2}$ for example 1

-... simulation results using first method

$* * * *$ theoretical results using eqn. 36

theoretical results using equ, 37

$a$ Against $\log k$
$b$ Against $\log \sigma_{c}^{2}$ 
using eqn. 28 for the second method, based on either the LS-SCORE or the cross-SCORE algorithms, are approximately given by

$S I N R_{2}=$

$\frac{M^{2} \rho_{s}^{-2}\left|1+\sigma_{c} c_{s}\right|^{2} S N R}{\sigma_{c}^{2}\left|c_{j}\right|^{2} M^{2} \rho_{j}^{-2} I N R+M \rho_{s}^{-2}\left(1+\sigma_{c} c_{s}+\sigma_{c} c_{s}^{*}\right)+\sigma_{c}^{2} \boldsymbol{a}_{e}^{H} \boldsymbol{R}_{z z}^{-2} \boldsymbol{a}_{e}}$

and

$$
\begin{aligned}
& E\left\{S I N R_{2}\right\} \\
& =\frac{M^{2} \rho_{s}^{-2} E\left\{\left|1+\sigma_{c} c_{s}\right|^{2}\right\} S N R}{\sigma_{c}^{2} E\left\{\left|c_{j}\right|^{2} M^{2} \rho_{j}^{-2} I N R+M \rho_{s}^{-2} E\left\{\left(1+\sigma_{c} c_{s}+\sigma_{c} c_{s}^{*}\right)\right\}\right.} \\
& +\sigma_{c}^{2} E\left\{\boldsymbol{a}_{e}^{H} \boldsymbol{R}_{z z}^{-2} \boldsymbol{a}_{e}\right\} \\
& =M^{2} \rho_{s}^{-2} S N R /\left[M \rho_{s}^{-2}+\left(M^{2} I N R+M\right) \rho_{j}^{-2}\right. \\
& \times \frac{M \sigma_{c}^{2}}{M^{2}-\left|\boldsymbol{a}_{j}^{H} \boldsymbol{a}_{d}\right|^{2}+M \sigma_{c}^{2}} \\
& \left.+\sigma_{c}^{2} \frac{(M-2)\left\{\left[k^{2} /\left(k+\sigma_{c}^{2}\right)^{2}\right] \sigma_{n}^{-4}\right\}\left(M^{2}-\left|\boldsymbol{a}_{j}^{H} \boldsymbol{a}_{d}\right|^{2}\right)}{M^{2}-\left|\boldsymbol{a}_{j}^{H} \boldsymbol{a}_{d}\right|^{2}+M \sigma_{c}^{2}}\right]
\end{aligned}
$$
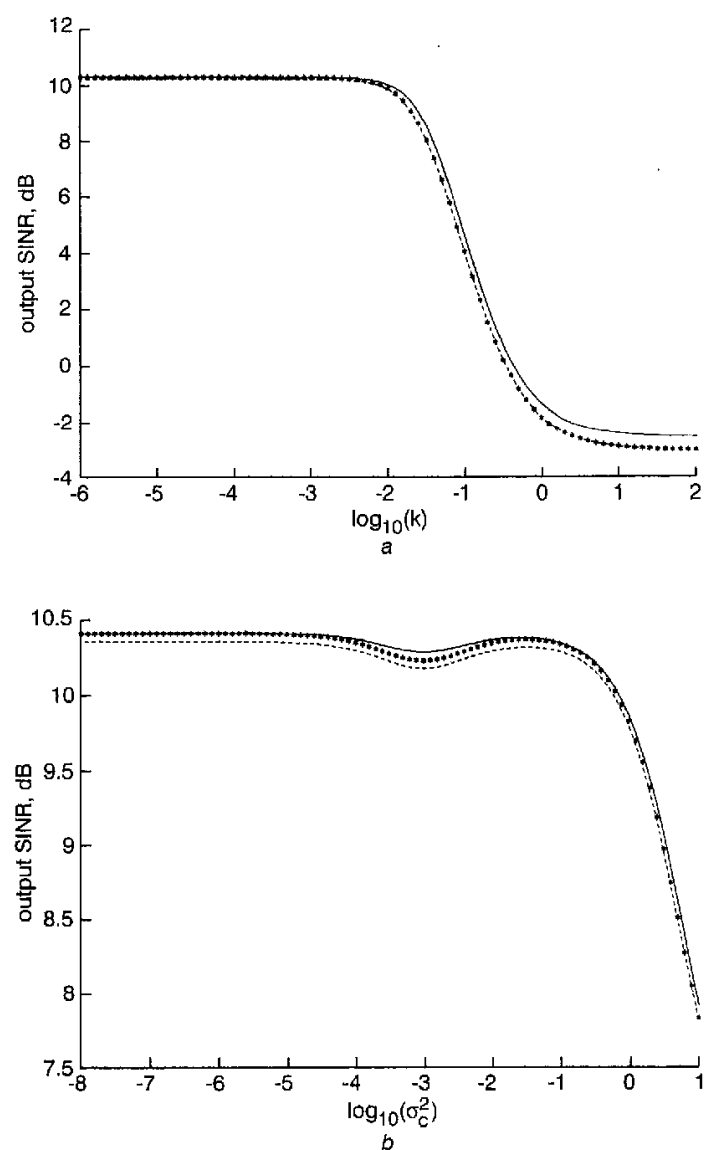

Fig. 2 Output SINR against logarithmic values of $k$ and $\sigma_{c}^{2}$ for example 2

-... simulation results using second method

$* * * *$ theoretical results using eqn. 38

theoretical results using eqn. 39

$a$ Against $\log k$

$b$ Against $\log \sigma_{0}^{2}$

IEE Proc.-Radar, Sonar Navig., Vol. 148, No. 4, August 200

\section{Computer simulations}

For all simulations, we use a ULA with $M=11$ and interelement spacing $=$ half wavelength of the SOI. A SOI impinging on the array from $5^{\circ}$ off broadside has $\mathrm{SNR}=0 \mathrm{~dB}$ and $\mathrm{CF}=2$, while an interference with direction angle $30^{\circ}$ off broadside has $\mathrm{INR}=9 \mathrm{~dB}$ and $\mathrm{CF}=3$. Moreover, both the SOI and interference are BPSK signals with rectangular pulse shape and baud rate $=0.1$. The sampling rate is set to 5 . The noise is spatially white Gaussian with mean zero and variance one. All the simulation results of the first four examples are obtained by averaging 1000 independent runs and using 2000 data snapshots for each run. We observe that 2000 data snapshots are enough for computing the correlation matrices required for the first four examples. 50 independent runs are averaged for showing the simulation results of the last example.

Example 1: Here, we confirm eqns. 36 and 37 when the optimal weight vector obtained by using the first method, based on either the LS-SCORE or the cross-SCORE algorithm, has the form of eqn. 28. Fig. 1 shows the array output SINR against the logarithmic value of $k$ with $\sigma_{c}^{2}=0.16$ and against the logarithmic value of $\sigma_{c}^{2}$ with $k=0.01$. We observe that the theoretical results
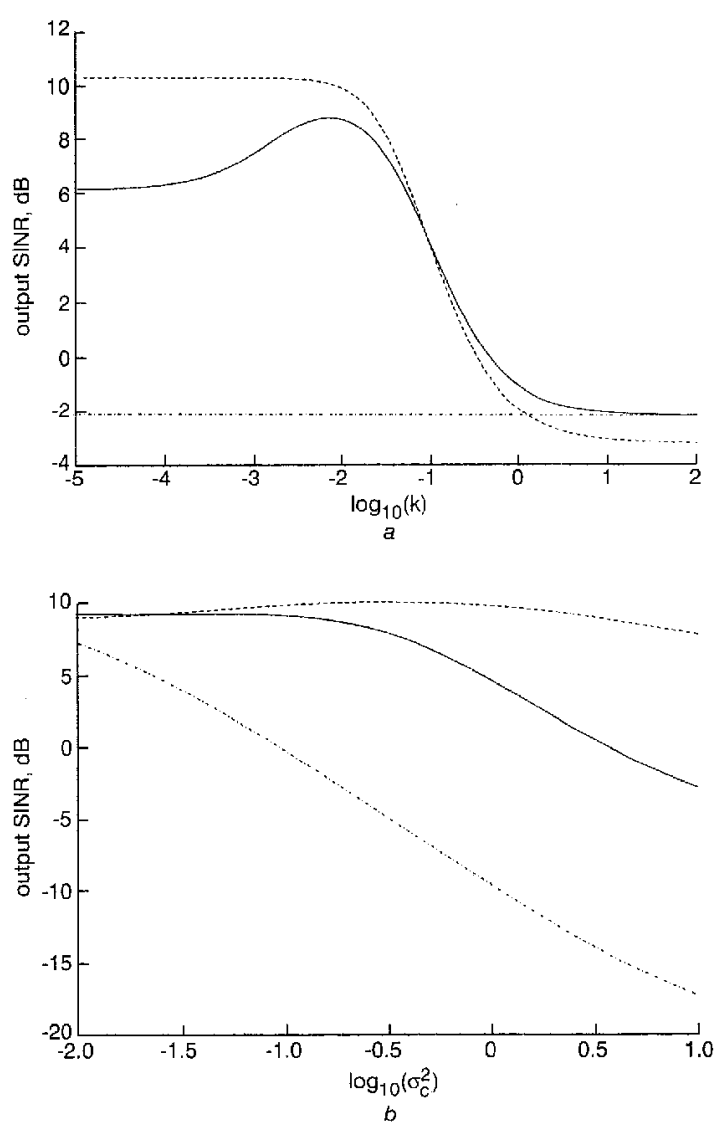

Fig. 3 Output SINR against logarithmic values of $k$ and $\sigma_{c}^{2}$ for example 3

-...- original LS-SCORE algorithm

- first method based on LS-SCORE algorithm

-.. second method based on LS-SCORE algorithm

$a$ Against $\log k$

$b$ Against $\log \sigma_{c}^{2}$ 
obtained by eqns. 36 and 37 are close to the simulation results. We also note that the mismatch between the theoretical and simulation results increases as $\sigma_{c}^{2} / k$ increases. This is mainly due to the fact that decreasing, $\sigma_{c}^{2} / k$ improves the approximation $\boldsymbol{a}_{j}^{H} \boldsymbol{E}_{s} \tilde{\boldsymbol{\Lambda}}_{s}^{-1} \boldsymbol{E}_{s}^{H} \boldsymbol{a}_{d} \approx 0$ for deriving eqn. 32 .

Example 2: Here, we confirm eqns. 38 and 39 when the optimal weight vector obtained by using the second method, based on either the LS-SCORE or the crossSCORE algorithm, has the form of eqn. 28. The output SINR against the logarithmic value of $k$ with $\sigma_{c}^{2}=0.16$, and against the logarithmic value of $\sigma_{c}^{2}$ with $k=0.001$, are plotted in Fig. 2. We observe that the theoretical results obtained by eqns. 38 and 39 are close to the simulation results.

Example 3: This example shows the effectiveness of the proposed methods based on the LS-SCORE algorithm. Substituting $\boldsymbol{R}_{x x}(\alpha, \tau)=r_{s s}(\alpha, \tau) \boldsymbol{a}_{d} \boldsymbol{a}_{d}^{H}$ into eqn. 11, we obtain $\boldsymbol{r}_{x r}(f)=\boldsymbol{R}_{x x}(f, \tau) c=r_{s s}(\alpha, \tau)\left(\boldsymbol{a}_{d}^{H} \boldsymbol{c}\right) \boldsymbol{a}_{d}+\boldsymbol{a}_{e}$ that has the form of $\boldsymbol{a}_{d}+\sigma_{c} \boldsymbol{a}_{e}$, where $\sigma_{c}=1 /\left[r_{s s}(\alpha, \tau)\left(\boldsymbol{a}_{d}^{H} \boldsymbol{c}\right)\right]$. Let $\sigma_{c} a_{e}$ be a white Gaussian random vector with mean zero and covariance matrix $\sigma_{c}^{2} I$. Fig. 3 depicts the output SINR against the logarithmic valuc of $k$ with $\sigma_{c}^{2}=0.16$ and
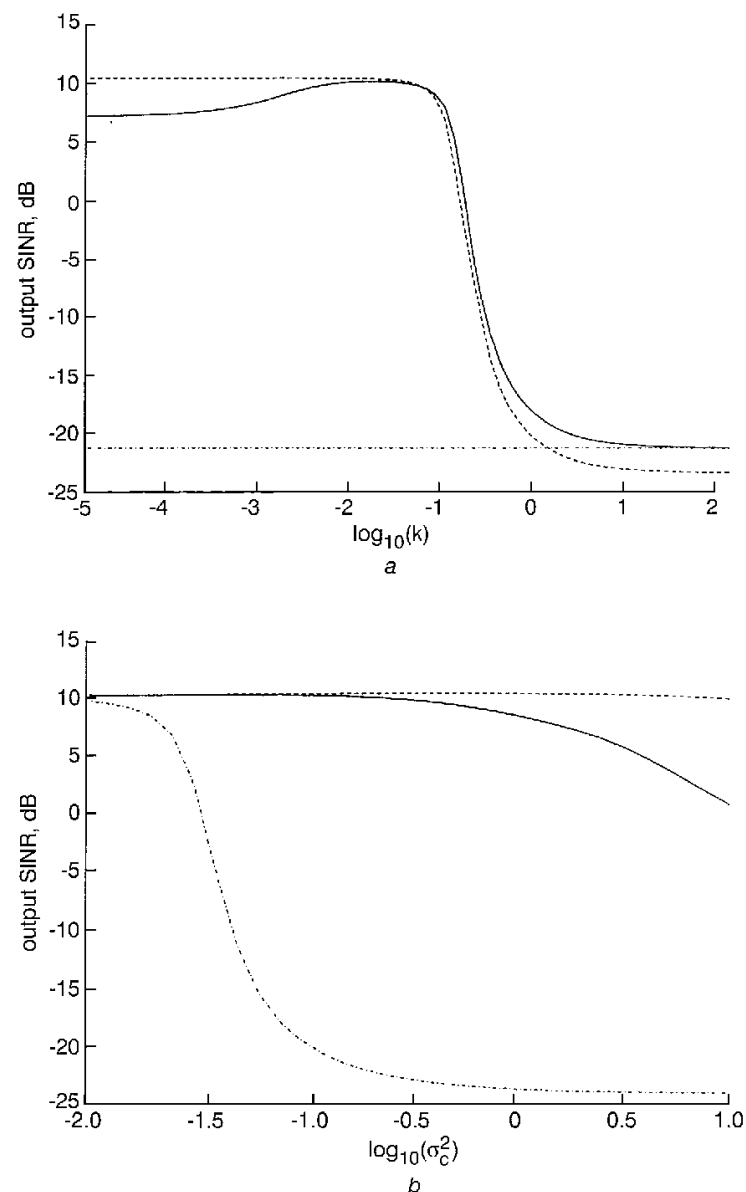

Fig. 4 Output SINR against logarithmic values of $k$ and $\sigma_{r}^{2}$ for exumple 4

-.-.-original cross-SCORE algorithm

- first method based on cross-SCORE algorithm

- . - second method based on cross-SCORE algorithm

a Against $\log k$

$b$ Against $\log \sigma_{c}^{2}$
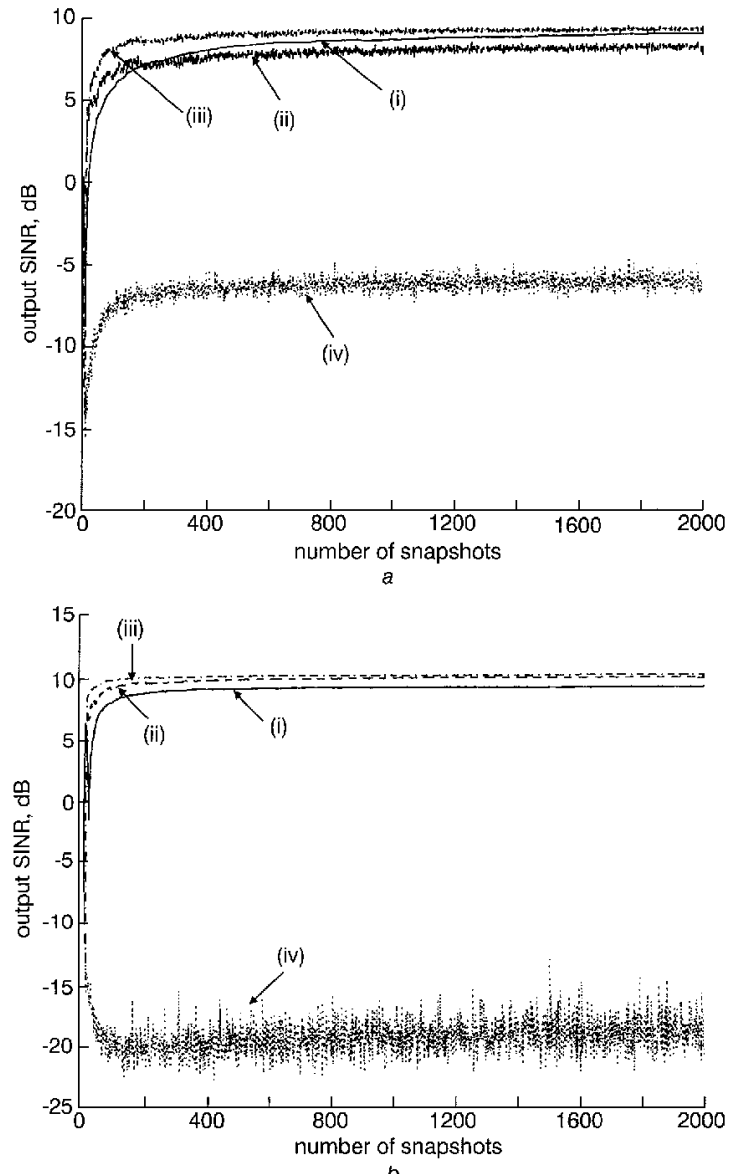

Fig. 5 Output SINR against number of snapshots for example 5 (i) Original al gorithm without $\mathrm{CFE}$

(ii) First method based on algorithm with random CFE

(iii) Second method based on algorithm with random $\mathrm{CHE}$

(iv) Original algorithm with random CFE

a LS-SCORL algorithm

$b$ Cross-SCORE algorithm

against the logarithmic value of $\sigma_{c}^{2}$ with $k=0.01$. The simulation results of the original LS-SCORE algorithm are also provided for comparison. We observe that the proposed methods based on the LS-SCORE algorithm can effectively alleviate the performance degradation due to the random CFE by choosing a suitable $k$. Fig. $3 b$ also shows that the second method is more effective than the first method, especially for large $\sigma_{c}^{2}$.

Example 4: Here, we show the effectiveness of the proposed methods based on the cross-SCORE algorithm. Substituting $\boldsymbol{R}_{x x}(\alpha, \tau)=r_{s s}(\alpha, \tau) \boldsymbol{a}_{d} \boldsymbol{a}_{d}^{H}$ into eqn. 10 , we obtain $\boldsymbol{R}_{x x}(f, \tau)=r_{s s}(\alpha, \tau) \boldsymbol{a}_{d} \boldsymbol{a}_{d}^{H}+\boldsymbol{R}_{e}(f, \tau)$ that has the form of $\boldsymbol{a}_{d} \boldsymbol{a}_{d}^{H}+\sigma_{c} \boldsymbol{R}_{e}(f, \tau)$, where $\sigma_{c}=1 /\left[r_{s s}(f, \tau)\right]$. Let the elements of $\boldsymbol{R}_{e}(f, \tau)$ be i.i.d. white Gaussian random variables with mean zero and variance one. The output SINR against the logarithmic value of $k$ with $\sigma_{c}^{2}=0.16$ and against the logarithmic value of $\sigma_{c}^{2}$ with $k=0.01$ is plotted in Fig. 4. We observe that the proposed methods provide satisfactory performance when $k$ is appropriately chosen. Fig. $4 b$ also shows that the second method is more robust than the first method, especially for large $\sigma_{c}^{2}$.

Example 5: We use the same SOI and interference as those used in the above four examples, except that an additional 
BPSK interferer with rectangular pulse shape, direction anglc $=40^{\circ}$ off broadside, INR $=6 \mathrm{~dB}, \mathrm{CF}=4$, and baud rate $=0.1$ is added. $k=0.01, \sigma_{c}^{2}=0.16$, and $C=\boldsymbol{I}$ are used by the proposed methods. Fig. 5 depict the output SINR against the number of data snapshots used. In comparison with the results of using the original SCORE algorithms, we observe that the proposed methods may provide better performance than the original SCORE algorithms, even in the presence of CFE. This is due to the fact that the term $\left(\sigma_{c}^{2} / k\right) I$ in eqn. 27 for the first method can reduce the finite sample effect if $\sigma_{c}^{2}$ is small and $k$ is appropriately set. The second method uses the eigenstructure of the autocorrelation matrix, and hence provides bettcr performance under a small $\sigma_{c}^{2}$ and suitable $k$.

\section{Conclusions}

This paper has shown that when the random CFE is an independent random sequence, the resulting cyclic correlation matrix equals the cyclic correlation matrix computed by using the actual cycle frequency plus a Gaussian random error matrix. Based on the theoretical result, two robust methods in conjunction with the SCORE algorithms have been developed to alleviate the performance deterioration duc to random CFE. Moreover, we have cvaluated the performance for each of the proposed methods. The validity of the theoretical work and the effectiveness of the proposed methods has also been demonstrated by simulation results.

\section{Acknowledgments}

This work was supported by the National Science Council under grant NSC88-2218-E002-027.

\section{References}

1 GARDNER, W.A.: 'Cyclostationarity in communications and signal processing” (New York, 1994)

2 AGEE, B.G., SCHFLL, S.V., and GARDNER, W.A.: 'Spectral self' coherence restoral: A new approach to blind adaptive signal extraction using antenna arrays', Proc. IEEE, 1990, 78, pp. 753-767

3 GARDNER, W. A.: 'Simplification of MUSIC and ESPRIT by exploitation of cyclostationarity', Proc. IEEE, 1988, 76, pp 845-847

4 SCHELL S.V. "Performance analysis of cyclic MUSIC method of direction estimation for cyclostationary signals', IEEE Trans. Signal direction estimation for cyclostationary

5 SCHELI, S.V.: Asymptotic moments of estimated cyclic correlation matrices', IEEE Trans. Signal Process., 1995, 43, (1), pp. 173-180

6 SCHEL, I, S.V., and GARDNER, W.A.: 'Progress on signal-selective direction finding'. Proccedinus of 5 th ASSP workshop on Spectrum estimation and modelling 1990 , Rochester. NY, pp. 144-148

7 BIEDKA T.F and AGEE B.G. 'Subinterval cyclic MUSIC - robust DF with error in cycle frequency knowledge'. Procecdings of 25 th
Asilomar Conference on Signals, systems and computers, 1991, Pacific Grove, CA, pp. 262-266

8 LEE, J.-H., and LEE, Y.-T.: "Robust adaptive array beamforming for cyclostationary signals under cycle frequency error', IEEE Trans. Antennas Propag., 1999, 47, pp. 233-241

9 I.EH, C.-C., and LEE, J.-H.: 'Robust adaptive array beamforming under steering vector errors', IEEE Trans. Antennas Propag., 1997, 45, pp. 168175

10 GIBRA, L.N.: 'Probability and statistical inference for scientists and engincers' (Prentice Hall, 1973)

\section{Appendix}

Since the clcments of $\boldsymbol{a}_{e}$ are white Gaussian random variables with mean zero and variance one, we have

$$
E\left\{c_{s}\right\}=E\left\{c_{j}\right\}=0
$$

because $E\left\{\boldsymbol{a}_{d}^{H} \boldsymbol{a}_{e}\right\}=E\left\{\boldsymbol{a}_{j}^{I I} \boldsymbol{a}_{e}\right\}=0$. Consequently:

$$
\begin{aligned}
E\left\{\left|c_{s}\right|^{2}\right\}= & \frac{1}{\left(M^{2}-\left|\boldsymbol{a}_{d}^{H} \boldsymbol{a}_{j}\right|^{2}\right)^{2}} \\
& \times\left\{M^{2} \boldsymbol{a}_{d}^{H} E\left\{\boldsymbol{a}_{e} \boldsymbol{a}_{e}^{H}\right\} \boldsymbol{a}_{d l}-M\left(\boldsymbol{a}_{j}^{H} \boldsymbol{a}_{d}\right) \boldsymbol{a}_{d}^{H} E\left\{\boldsymbol{a}_{e} \boldsymbol{a}_{e}^{I \prime}\right\} \boldsymbol{a}_{j}\right. \\
& \left.-M\left(\boldsymbol{a}_{d l}^{H} \boldsymbol{a}_{j}\right) \boldsymbol{a}_{j}^{H} E\left\{\boldsymbol{a}_{e} \boldsymbol{a}_{e}^{H}\right\} \boldsymbol{a}_{d}+\left|\boldsymbol{a}_{d}^{I I} \boldsymbol{a}_{j}\right|^{2} \boldsymbol{a}_{j}^{I I} E\left\{\boldsymbol{a}_{e} \boldsymbol{a}_{e}^{H}\right\} \boldsymbol{a}_{j}\right\} \\
= & \frac{M}{M^{2}-\left|\boldsymbol{a}_{d}^{H} \boldsymbol{a}_{j}\right|^{2}}
\end{aligned}
$$

where $E\left\{\boldsymbol{a}_{e} \boldsymbol{a}_{e}^{H}\right\}=\boldsymbol{I}$. Similarly, we have

$$
E\left\{\left|c_{j}\right|^{2}\right\}=\frac{M}{M^{2}-\left|\boldsymbol{a}_{d}^{H} \boldsymbol{a}_{j}\right|^{2}}
$$

Next, we substitute $\boldsymbol{a}_{e}=c_{s} \boldsymbol{a}_{d}+c_{j} \boldsymbol{a}_{j}+\boldsymbol{E}_{n} \boldsymbol{E}_{n}^{H} \boldsymbol{a}_{e}$ into $E\left\{\boldsymbol{a}_{e}^{H} \boldsymbol{R}_{z z}^{-2} \boldsymbol{a}_{e}\right\}$ and obtain

$$
\begin{aligned}
E\left\{\boldsymbol{a}_{e}^{H} \boldsymbol{R}_{z z}^{-2} \boldsymbol{a}_{e}\right\} \approx & E\left\{\left|c_{s}\right|^{2}\right\} \boldsymbol{a}_{d}^{H} \boldsymbol{E}_{s} \tilde{\boldsymbol{\Lambda}}_{s}^{-2} \boldsymbol{E}_{s}^{H} \boldsymbol{a}_{d} \\
& +E\left\{\left|c_{j}\right|^{2}\right\} \boldsymbol{a}_{j}^{H} \boldsymbol{E}_{s} \tilde{\boldsymbol{\Lambda}}_{s}^{-2} \boldsymbol{E}_{s}^{H} \boldsymbol{a}_{j} \\
& +E\left\{\boldsymbol{a}_{e}^{H} \boldsymbol{E}_{n} \tilde{\boldsymbol{\Lambda}}_{n}^{-2} \boldsymbol{E}_{n}^{H} \boldsymbol{a}_{e}\right\}
\end{aligned}
$$

It is easy to show that

$$
E\left\{\boldsymbol{a}_{e}^{H} \boldsymbol{E}_{n} \tilde{\boldsymbol{\Lambda}}_{n}^{-2} \boldsymbol{E}_{n}^{H} \boldsymbol{a}_{e}\right\}=\operatorname{trace}\left\{\tilde{\boldsymbol{\Lambda}}_{n}^{-2}\right\}=(M-2)\left(\sigma_{n}^{2}+\frac{\sigma_{c}^{2}}{k}\right)^{-2}
$$

when using the first method, and

$$
E\left\{\boldsymbol{a}_{e}^{H} \boldsymbol{E}_{n} \tilde{\boldsymbol{\Lambda}}_{n}^{-2} \boldsymbol{E}_{n}^{H} \boldsymbol{a}_{e}\right\}=\operatorname{trace}\left\{\tilde{\boldsymbol{\Lambda}}_{n}^{-2}\right\}=(M-2) \frac{k^{2}}{\left(k+\sigma_{e}^{2}\right)^{2}} \sigma_{n}^{-4}
$$

when using the second method. 